\title{
Proton incorporations and superconductivity in a cobalt oxyhydrate
}

\author{
Guanghan $\mathrm{Cao}^{\mathrm{a}, *}$, Xiaoming Tang ${ }^{\mathrm{b}}$, Yi Xu ${ }^{\mathrm{a}}$, Ming Zhong ${ }^{\mathrm{a}}$, \\ Xuezhi Chen ${ }^{a}$, Chunmu Feng ${ }^{b}$, and Zhu'an $\mathrm{Xu}^{\mathrm{a}}$ \\ ${ }^{a}$ Department of Physics, Zhejiang University, Hangzhou, Zhejiang 310027, \\ People's Republic of China \\ ${ }^{\mathrm{b}}$ Test and Analysis Center, Zhejiang University, Hangzhou, Zhejiang 310027, \\ People's Republic of China
}

\begin{abstract}
We report the evidence of proton incorporations in a newly-discovered cobalt oxyhydrate superconductor. During the hydration process for $\mathrm{Na}_{0.32} \mathrm{CoO}_{2}$ by the direct reaction with water liquid, it was shown that substantial $\mathrm{NaOH}$ was gradually liberated, indicating that $\mathrm{H}^{+}$is incorporated into the hydrated compound. Combined with the thermogravimetric analysis, the chemical composition of the typical sample is $\mathrm{Na}_{0.22} \mathrm{H}_{0.1} \mathrm{CoO}_{2} \cdot 0.85 \mathrm{H}_{2} \mathrm{O}$, which shows bulk superconductivity at $4.4 \mathrm{~K}$.
\end{abstract}

Key words:

PACS: 74.62.Bf; 74.70.-b;74.10.+v

A. Superconductors, C. Thermogravimetric analysis; C. X-ray diffraction

Recently, Takada et al. [1] discovered the first cobalt oxide superconductor denoted as $\mathrm{Na}_{x} \mathrm{CoO}_{2} \cdot y \mathrm{H}_{2} \mathrm{O}(x \approx 0.35, y \approx 1.3)$. Though the superconducting transition temperature $T_{c}(\sim 5 \mathrm{~K})$ is not striking, the underlying physics is so attractive that many research groups have been following this topic $[2,3,4,5,6,7,8,9,10,11,12,13,14,15,16,17]$. Various audacious ideas and predictions have been proposed by the theorists $[2,3,4,5,6]$, waiting for the experimental verifications. On the other hand, experimental studies were found to be very difficult primarily due to the extreme chemical instability of the compound [7]. Inconsistent experimental results often appear in the literatures. Therefore, it is essential to carefully characterize the sample before the physical, structural, and spectral properties are measured. Up to present, the chemical characterizations of the sample mainly focus on the sodium and

\footnotetext{
* Corresponding author

Email address: ghcao@zju.edu.cn (Guanghan Cao).
} 


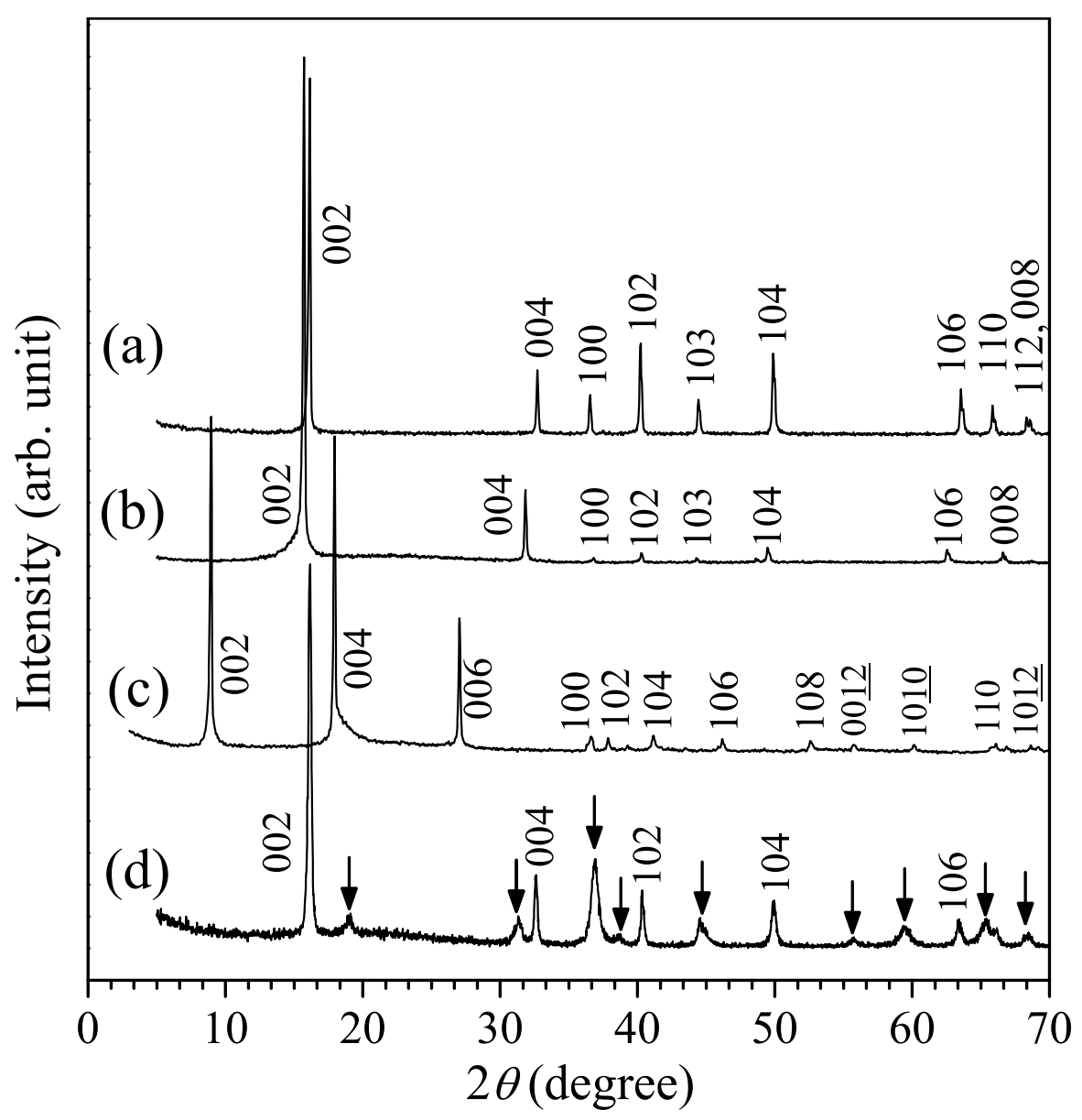

Fig. 1. X-ray diffraction patterns of (a) $\mathrm{Na}_{0.7} \mathrm{CoO}_{2}$, (b) $\mathrm{Na}_{0.32} \mathrm{CoO}_{2}$, (c) the water-liquid-hydrated (WLH) sample, and (d) as-fired WLH sample at $673 \mathrm{~K}$ in air for 0.5 hours. The diffraction indices for the peaks are labelled. In the pattern $(\mathrm{d})$, the peaks marked by arrows indicate that $\mathrm{Co}_{3} \mathrm{O}_{4}$ is separated out.

water content $[8,9,10,11,12]$. In this paper, we report the evidence of proton incorporations in the new superconducting cobalt oxyhydrate.

Similar to the previous reports [1,7], samples were prepared in the following steps. First, $\mathrm{Na}_{0.7} \mathrm{CoO}_{2}$ polycrystals were synthesized by three rounds of fast solid-state reaction at $1083 \mathrm{~K}$ in flowing oxygen with two intermediate regrindings, using $\mathrm{Na}_{2} \mathrm{CO}_{3}(99.9 \%)$ and $\mathrm{Co}_{3} \mathrm{O}_{4}(99.99 \%)$ as the starting materials. Although the original composition is $\mathrm{Na}_{0.74} \mathrm{CoO}_{2}$, the actual composition was determined to be $\mathrm{Na}_{0.7} \mathrm{CoO}_{2}$ by the atomic absorption spectroscopy (AAS) and the $\mathrm{Na}^{+}$ion-selective electrode (ISE) techniques. The loss of sodium is primarily due to the volatilization of $\mathrm{Na}_{2} \mathrm{O}$ during the solid-state reaction. Fig. 1(a) shows the powder x-ray diffraction (XRD) pattern for $\mathrm{Na}_{0.7} \mathrm{CoO}_{2}$, as measured with $\mathrm{Cu} \mathrm{K} \alpha$ radiation. All the diffraction peaks can be well indexed using a hexagonal cell with $a=2.831 \AA$ and $c=10.918 \AA$.

In the second step, $\mathrm{Na}_{0.7} \mathrm{CoO}_{2}$ was oxidized by the excessive bromine dissolved 
in acetonitrile. This process resulted in the deintercalation of the sodium, producing the thermodynamically metastable hexagonal phase $\mathrm{Na}_{x} \mathrm{CoO}_{2}(0.25<$ $x<0.7)$. The value of $x$ depends on the equilibrium concentrations of $\mathrm{Br}_{2}$, $\mathrm{Br}^{-}$, and $\mathrm{Na}^{+}$, provided the reaction time is long enough (over 48 hours for polycrystalline samples). In the case of the sample preparation in the present study, $2.140 \mathrm{~g} \mathrm{Na}_{0.7} \mathrm{CoO}_{2}$ reacted with $3.70 \mathrm{~g} \mathrm{Br}_{2}$ dissolved in $10.0 \mathrm{ml}$ acetonitrile in a closed container at $310 \mathrm{~K}$ for 48 hours. After the reaction completed, the solid product was washed several times with acetonitrile and then dried in vacuum. The mass of the solid $\mathrm{Na}_{x} \mathrm{CoO}_{2}$ became $1.965 \mathrm{~g}$. From the loss of mass, one can estimate that the $x=0.32$, which is in very good agreement with our ISE measurement result. Fig. 1(b) shows the XRD pattern for the sample $\mathrm{Na}_{0.32} \mathrm{CoO}_{2}$. It has the same crystal structure with that of $\mathrm{Na}_{0.7} \mathrm{CoO}_{2}$, but the unit cell becomes elongated: $a=2.811 \AA$ and $c=11.211 \AA$. The shrinkage of $a$-axis is ascribed to the increase of the oxidation state of cobalt, and the stretch of $c$-axis is due to the relatively weak Coulomb attractions along the $c$-axis when $\mathrm{Na}^{+}$is partially deintercalated.

The intermediate compound $\mathrm{Na}_{0.32} \mathrm{CoO}_{2}$ easily absorbs water. So, most literatures employed the reaction with water vapor for the hydration. However, deliquescence often happens, making it difficult to distinguish between crystal water and free water. Now that free water inclusion is inevitable, one can prepare the hydrated compound by the direct reaction with water liquid or solution, and it was proved to be successful $[13,14]$. In this study, we employed both routes to synthesize the hydrated compound in order to make a comparison. Partial $\mathrm{Na}_{0.32} \mathrm{CoO}_{2}$ sample reacted with water vapor at room temperature for one week, obtaining the water-vapor-hydrated (WVH) compound. Another part of $\mathrm{Na}_{0.32} \mathrm{CoO}_{2}$ was soaked with water liquid in a closed container at room temperature for one week. This product is hereafter called waterliquid-hydrated (WLH) sample. XRD patterns of the two hydrated products are quite similar. By the least-squared fitting, the cell parameters were calculated as $a=2.823 \AA$ and $c=19.61 \AA$ for the WVH compound, consistent with the previous report [1]. However, the cell parameters for the WLH product are $a=2.824 \AA$ and $c=19.75 \AA$. The obvious difference in the value of $c$-axis implies some minute change in the crystal structure. We will discuss this issue later. Fig. 1(c) shows the XRD pattern of the WLH sample, which indicates that it is a hexagonal single phase.

The thermal instability of samples was investigated by using a thermal analyzer which can simultaneously measure the temperature dependence of weight (thermogravimetric analysis, TGA) and the temperature difference between the sample and the reference (differential thermal analysis, DTA). The experiments were performed under ambient condition ( $T=298 \mathrm{~K}$, humidity: $\sim 50 \%$ ) using the sweep rate of $20 \mathrm{~K} / \mathrm{min}$. Fig. 2 shows the TGA result for the different samples. The first curve for $\mathrm{Na}_{0.7} \mathrm{CoO}_{2}$ shows an abrupt loss of weight at $1300 \mathrm{~K}$, accompanied with a big endothermal peak in the DTA curve (not 


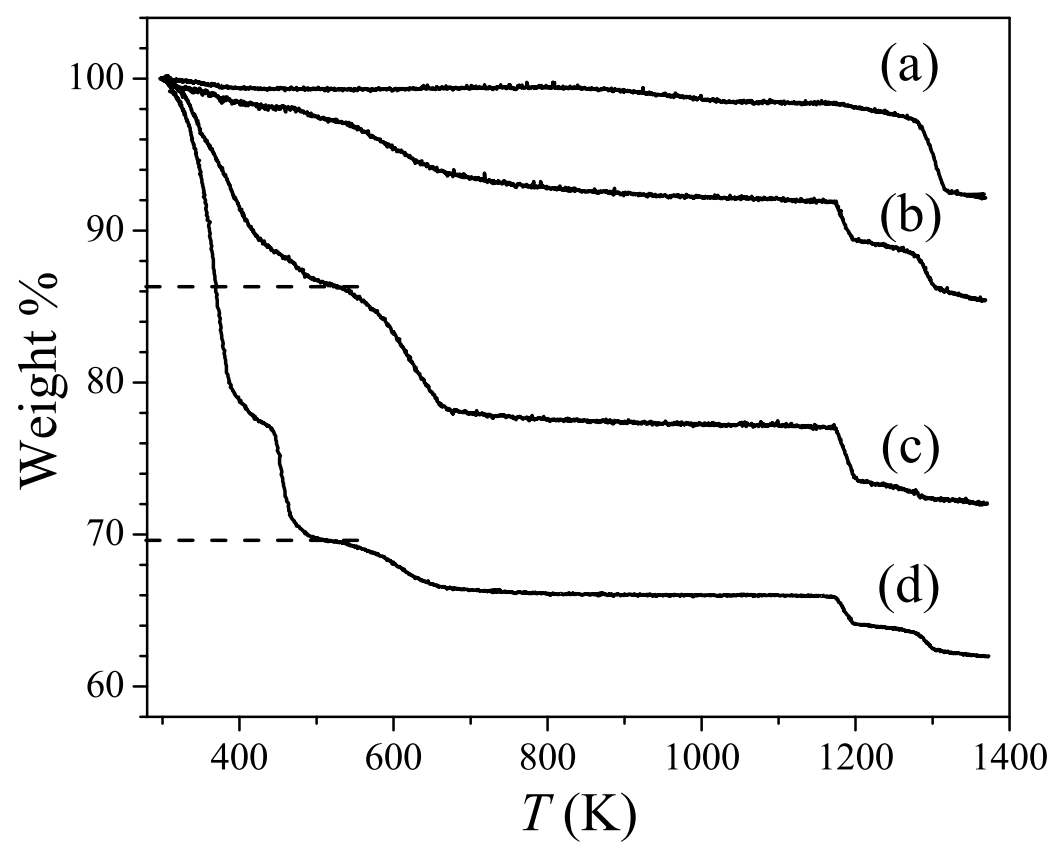

Fig. 2. Thermogravimetric curves of (a) $\mathrm{Na}_{0.7} \mathrm{CoO}_{2}$, (b) $\mathrm{Na}_{0.32} \mathrm{CoO}_{2}$, (c) the water-liquid-hydrated compound, and $(\mathrm{d})$ the water-vapor-hydrated compound.

shown here). The measured sample was found to have molten after cooling down. Therefore, the decomposition can be expressed as

$$
\mathrm{Na}_{0.7} \mathrm{CoO}_{2} \longrightarrow \operatorname{Liquid}\left(0.35 \mathrm{Na}_{2} \mathrm{O}+\mathrm{CoO}\right)+0.325 \mathrm{O}_{2} \uparrow \text {. }
$$

In the second curve for $\mathrm{Na}_{0.32} \mathrm{CoO}_{2}$, there are weight-losses at $605 \mathrm{~K}, 1184 \mathrm{~K}$, and $1291 \mathrm{~K}$, respectively. XRD measurement for the sample annealed at 673 $\mathrm{K}$ in air shows that $\mathrm{Co}_{3} \mathrm{O}_{4}$ is separated out, like the case in Fig. 1(d). So, the loss of weight at $\sim 605 \mathrm{~K}$ corresponds to the following equation,

$$
\mathrm{Na}_{0.32} \mathrm{CoO}_{2} \longrightarrow 0.457 \mathrm{Na}_{0.7} \mathrm{CoO}_{2}+0.181 \mathrm{Co}_{3} \mathrm{O}_{4}+0.181 \mathrm{O}_{2} \uparrow \text {. }
$$

Since $\mathrm{Co}_{3} \mathrm{O}_{4}$ decomposes at about $1180 \mathrm{~K}$ in air, the weight-loss at $1184 \mathrm{~K}$ is ascribed to the decomposition of $\mathrm{Co}_{3} \mathrm{O}_{4}$,

$$
\mathrm{Co}_{3} \mathrm{O}_{4} \longrightarrow 3 \mathrm{CoO}+0.5 \mathrm{O}_{2} \uparrow \text {. }
$$

It is noted that the theoretical weight-losses based on Eqs. (1), (2) and (3) are basically consistent with the experimental results.

As for the hydrated samples, heavy weight-loss was observed below $518 \mathrm{~K}$, which is due to the loss of water. Around $600 \mathrm{~K}$, the weight-loss is associated with the decomposition of the metastable $\mathrm{Na}_{x} \mathrm{CoO}_{2}$, as indicated by Fig. 1(d). 
The other two weight-losses at higher temperatures can be described by the Eqs. (1) and (3), respectively. Since the sodium content was determined to be 0.22 for the WLH sample (see below), the water content for this compound is thus estimated to be 0.85 . This value is obviously lower than the common value $\sim 1.3[1,7,9,12]$. On the contrary, the water content for the WVH compound is determined as 2.3 , which is remarkably higher than that of previous reports. We suspect that free water had been absorbed in the WVH sample. It is noted that the ratio of weight-loss at $1184 \mathrm{~K}$ and $1291 \mathrm{~K}$ is quite different for the two hydrated samples. This is because that the sodium content in the WLH compound is lower than that in the WVH sample.

The sodium content in the cobaltates was measured by AAS and $\mathrm{Na}^{+}$ISE techniques, respectively. In the AAS method, the sample was dissolved in 2 mol/L $\mathrm{HNO}_{3}$ solution and then diluted into appropriate concentrations for the measurement. Blank data (parallel experiment result with no sample dissolved) were collected and then deducted because the $\mathrm{Na}$ concentration in the $\mathrm{HNO}_{3}$ solution is generally not neglectable. In the ISE measurement, the sample was dissolved in acid solution, and then the solution was neutralized by $\left(\mathrm{CH}_{3}\right)_{2} \mathrm{CHNH}_{2}$ to eliminate the disturbance of $\mathrm{H}_{3} \mathrm{O}^{+}$. Both measurements show that the sodium content for the WLH compound is $0.22(1)$, which is $1 / 3$ smaller than that of the WVH one.

We also used the ISE technique to determine the $\mathrm{Na}^{+}$concentration dynamically. Fig. 3 shows the change of $\mathrm{Na}^{+}$concentration against the time of the hydration reaction at $300 \mathrm{~K}$. It can be seen that the liberated $\mathrm{Na}^{+}$increases with increasing the reaction time. During the first 10 hours of the hydration, $\mathrm{Na}^{+}$concentration increases rapidly. Then, it increases gradually until the saturation at $0.01 \mathrm{~mol} / \mathrm{L}$ when $t=120$ hours. It was also noted that the $\mathrm{pH}$ value increases from 9.0 at the beginning of the hydration to 12.0 when the reaction completed. That is to say, $\mathrm{NaOH}$ is gradually liberated during the hydration reaction. Considered that the hydration is not a redox reaction, therefore, $\mathrm{H}^{+}$ has to be incorporated in the hydrated product. By using the above TGA result, the WLH compound can be expressed as $\mathrm{Na}_{0.22} \mathrm{H}_{0.1} \mathrm{CoO}_{2} \cdot 0.85 \mathrm{H}_{2} \mathrm{O}$, and the hydration can be described as

$$
\mathrm{Na}_{0.32} \mathrm{CoO}_{2}+0.95 \mathrm{H}_{2} \mathrm{O} \longrightarrow \mathrm{Na}_{0.22} \mathrm{H}_{0.1} \mathrm{CoO}_{2} \cdot 0.85 \mathrm{H}_{2} \mathrm{O}+0.1 \mathrm{Na}^{+}+0.1 \mathrm{OH}^{-} \text {. }
$$

Apparently, the equation above consists of the ion-exchange of $\mathrm{Na}^{+}$and $\mathrm{H}^{+}$. So, it is not strange that the content of the incorporated proton was found to alter with the change of the concentration of $\mathrm{NaOH}$ in the reactor. When the $\mathrm{NaOH}$ was removed, the content of the incorporated proton could increase up to 0.15 . On the contrary, in the case of high $\mathrm{NaOH}$ concentration, the amount of the incorporated $\mathrm{H}^{+}$will be reduced. Note that free water may exist in the WVH sample, we speculate that small amount of proton might be also incorporated in the $\mathrm{Na}_{0.32} \mathrm{CoO}_{2} \cdot 2.3 \mathrm{H}_{2} \mathrm{O}$ phase. 


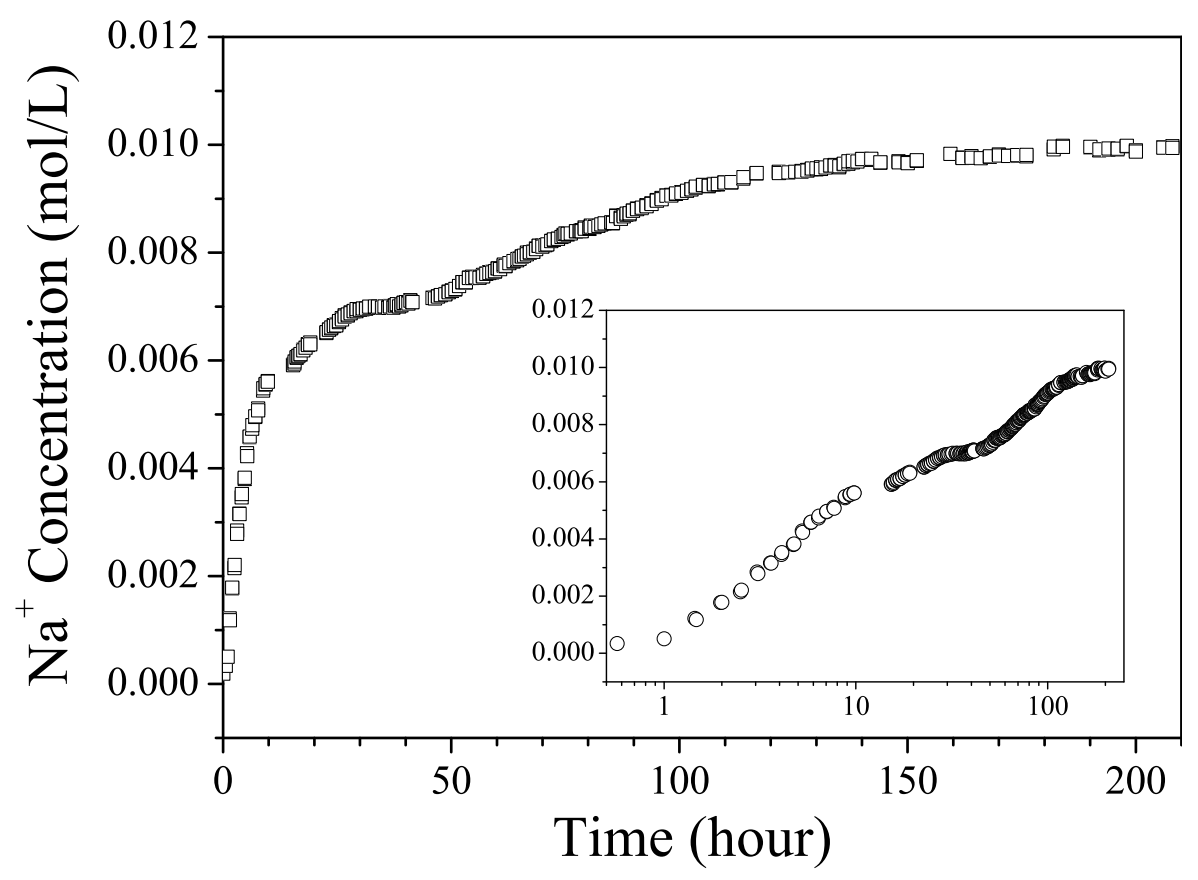

Fig. 3. $\mathrm{Na}^{+}$concentration as a function of the hydration reaction time at $300 \mathrm{~K}$. Note that the inset uses the logarithm scale for the horizontal axis.

Now, let us discuss the possible crystal structure of $\mathrm{Na}_{0.22} \mathrm{H}_{0.1} \mathrm{CoO}_{2} \cdot 0.85 \mathrm{H}_{2} \mathrm{O}$. As we know, the anhydrus parent compound $\mathrm{Na}_{0.32} \mathrm{CoO}_{2}$ consists of triangular $\mathrm{CoO}_{2}$ layers in which cobalt is octahedrally coordinated. Partially-occupied $\mathrm{Na}^{+}$is sandwiched by the $\mathrm{CoO}_{2}$ layers. Water can be intercalated between $\mathrm{CoO}_{2}$ layers and $\mathrm{Na}^{+}$layers when the sodium is deintercalated to some extent. By using neutron diffractions, detailed structural models were established $[15,16]$. Impressively, Jorgensen et. al. [16] proposed that the position of $\mathrm{Na}^{+}$was shifted in such a way to accommodate the tetrahedral coordinations by water molecules. In this model, the ideal $\mathrm{Na}$ to $\mathrm{H}_{2} \mathrm{O}$ ratio is 1:4, which also satisfies the chemical formula $\mathrm{Na}_{0.22} \mathrm{H}_{0.1} \mathrm{CoO}_{2} \cdot 0.85 \mathrm{H}_{2} \mathrm{O}$. So, we think that similar structure is probable for the present WLH compound. Another problem concerns about the position of the incorporated $\mathrm{H}^{+}$. From chemical bonding point of view, $\mathrm{H}^{+}$may bond with the oxygen in $\mathrm{H}_{2} \mathrm{O}$ to form $\mathrm{H}_{3} \mathrm{O}^{+}$, or, bond with the oxygen in $\mathrm{CoO}_{2}$ layers to form the $\mathrm{OH}$ groups. We have measured the IR spectra, but no definite conclusion can be drawn about the bonding of the $\mathrm{H}^{+}$.

The longer $c$-axis of $\mathrm{Na}_{0.22} \mathrm{H}_{0.1} \mathrm{CoO}_{2} \cdot 0.85 \mathrm{H}_{2} \mathrm{O}$ compared with that of $\mathrm{Na}_{0.32} \mathrm{CoO}_{2}$. $2.3 \mathrm{H}_{2} \mathrm{O}$ can be explained in terms of Coulomb attractions between the $\mathrm{Na}^{+}$ layers and $\mathrm{CoO}_{2}$ layers. One would expect that relatively weak Coulomb attractions along the $c$-axis for $\mathrm{Na}_{0.22} \mathrm{H}_{0.1} \mathrm{CoO}_{2} \cdot 0.85 \mathrm{H}_{2} \mathrm{O}$ due to the relatively less electric charge in the $\mathrm{Na}^{+}$and $\mathrm{CoO}_{2}$ layers. So, though the water content is not high in $\mathrm{Na}_{0.22} \mathrm{H}_{0.1} \mathrm{CoO}_{2} \cdot 0.85 \mathrm{H}_{2} \mathrm{O}$, the $c$-axis parameter can be even larger than that of $\mathrm{Na}_{0.32} \mathrm{CoO}_{2} \cdot 2.3 \mathrm{H}_{2} \mathrm{O}$. 


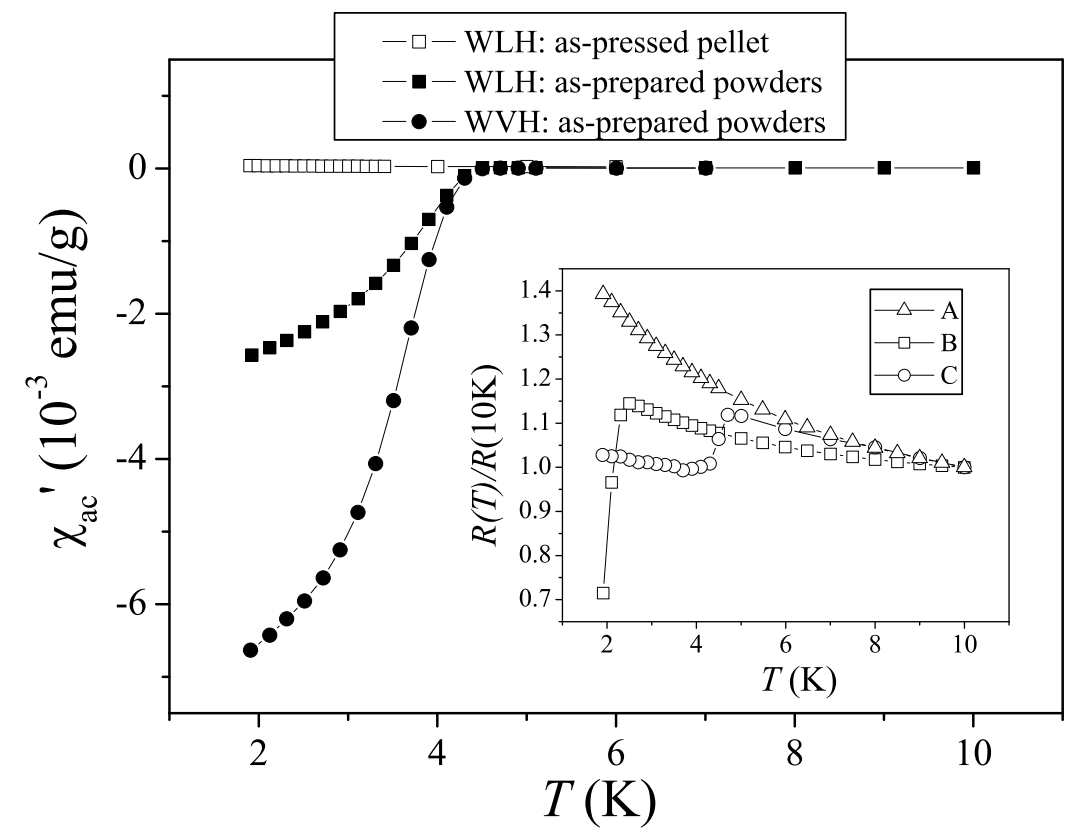

Fig. 4. Superconducting transitions measured by ac susceptibility and dc resistance. In the inset, data A was collected in a fresh pellet pressed with the pressure of 5000 $\mathrm{kg} / \mathrm{cm}^{2}$. Data B and C were measured after the pellet was placed in humid conditions at room temperature for one day and two weeks, respectively.

The superconducting transition was investigated on a Quantum Design PPMS system. As shown in Fig. 4, the as-prepared WVH and WLH samples shows diamagnetic transition at $4.5 \mathrm{~K}$ and $4.4 \mathrm{~K}$, respectively. The sharp transitions indicate that both samples have bulk superconductivity, though the diamagnetic signal for the sample WVH is stronger. This implies that the proton incorporation does not influence the superconductivity so much.

It was found that the superconductivity in $\mathrm{Na}_{0.22} \mathrm{H}_{0.1} \mathrm{CoO}_{2} \cdot 0.85 \mathrm{H}_{2} \mathrm{O}$ is very sensitive to the details of the measurement operations. While the as-prepared WLH powders show bulk superconductivity at $4.4 \mathrm{~K}$, the as-pressed pellets (with the pressure of $5000 \mathrm{~kg} / \mathrm{cm}^{2}$ ) shows no superconducting transition above 1.9 K. The resistance measurement shown in the inset of Fig. 4 confirms the result in an opposite way. The fresh pellet shows no superconducting transition above $1.9 \mathrm{~K}$, however, after the very same sample was placed in humid environment at room temperature over a period of time, superconductivity was recovered. Moreover, $T_{c}$ increases with increasing the placement time. Therefore, it should be careful to draw any conclusions on the relationship between $T_{c}$ and the factors, such as water and sodium content. We checked the XRD patterns for the as-pressed and the as-placed pellets. Both data indicates single phase of $c=19.7 \AA$, however, the as-pressed sample shows very broad diffraction peaks, and the broad peaks change back into sharp ones for 
the as-placed sample. This observation suggests that the crystallinity, affected by water, be important for the superconductivity.

In summary, the water-liquid-hydrated cobaltate sample, which shows bulk superconductivity, was carefully characterized by the XRD, ISE, TGA measurements. Evidence of proton incorporations has been given, though the position and the chemical bonding of the hydrogen ions are not clear. It was observed that the superconductivity is extremely sensitive to temperature, pressure and humidity. Crystallinity seems to play a role in the appearance of superconductivity.

\section{Acknowledgements}

This work was supported by NSFC under Grant No. 10104012 and 10225417. Partial support under Project No. NKBRSF-G1999064602 is also acknowledged. 


\section{References}

[1] K. Takada, H. Sakurai, E. Takayama-Muromachi, F. Izumi, R. A. Dilanian, T. Sasaki, Nature 422 (2003)53.

[2] G. Baskaran, Phys. Rev. Lett. 91 (2003) 097003.

[3] B. Kumar and B. S. Shastry, Phys. Rev. B 68 (2003) 104508.

[4] Q. H. Wang, D. H. Lee, and P. A. Lee, cond-mat/0304377.

[5] A. Tanaka and X. Hu, Phys. Rev. Lett. 91 (2003) 257006.

[6] Y. Tanaka, Y. Yanase, M. Ogata, cond-mat/0311266.

[7] M. L. Foo, R. E. Schaak, V. L. Miller, T. Klimczuk, N. S. Rogado, Y. Wang, G. C. Lau, C. Cralet, H. W. Zandbergen, N. P. Ong, R. J. Cava, Solid State Commun. 127 (2003) 33.

[8] R. E. Schaak, T. Klimczuk, M. L. Foo, R. J. Cava, Nature 424 (2003) 527.

[9] J. Cmaidalka, A. Baikalov, Y. Y. Xue, R. L. Meng, C. W. Chu, cond-mat/0308301

[10] H. Sakurai, K. Takada, F. Izumi, D. A. Dilanian, T. Sasaki, E. TakayamaMuromachi, cond-mat/0310717.

[11] C. J. Milne, D. N. Argyriou, A. Chemseddine, N. Aliouane, J. Veira, D. Alber, cond-mat/0401273.

[12] D. P. Chen, H. C. Chen, A. Maljuk, A. Kulakov, H. Zhang, C. T. Lin, cond-mat/0401636

[13] G. Cao, C. Feng, Y. Xu, W. Lu, J. Shen, M. Fang, Z. Xu, J. Phys.: Condens. Matter 15 (2003) L519.

[14] S. Park, Y. Lee, A. Moodenbaugh, T. Vogt, Phys. Rev. B 68 (2003) 180505.

[15] J. W. Lynn, Q, Huang, C. M. Brwon, V. L. Miller, M. L. Foo, R. E. Schaak, C. Y. Jones, E. A. Mackey, R. J. Cava, Phys. Rev. B 68 (2003) 214516.

[16] J. D. Jorgensen, M. Avdeev, D. G. Hinks, J. C. Burley, S. Short, Phys. Rev. B 68 (2003) 214517.

[17] R. Jin, B. C. Sales, P. Khalifah, D. Mandrus, Phys. Rev. Lett. 91 (2003) 217001. 